INVESTIGACIONES

\title{
EL DESARROLLO MORAL EN EL DEPORTE ESCOLAR EN EL CONTEXTO EUROPEO: UN ESTUDIO BASADO EN DILEMAS SOCIOMORALES
}

\author{
THE MORAL DEVELOPMENT IN SCHOOL SPORTS IN THE EUROPEAN CONTEXT: \\ A STUDY BASED ON SOCIAL-MORAL DILEMMAS \\ O desenvolvimento moral do esporte escolar no contexto Europeu: \\ um estudo baseado em dilemas socio-morais
}

\section{Antonio Fraile Aranda}

Doctor en Ciencias de la Educación Facultad de Educación y Trabajo Social. Paseo Belén $\mathrm{N}^{\circ}$ 1. 47011. Valladolid afraile@mpc.uva.es

\begin{abstract}
RESUMEN
El deporte escolar es una actividad educativa de gran participación en el contexto nacional e internacional. Este es un estudio sobre el desarrollo moral, a través de dilemas sociomorales, con escolares de 12 años que participan en el deporte escolar en el contexto europeo de: España, Francia, Italia y Portugal. Se emplea una escala de valoración de cada uno de los dilemas y un grupo de discusión en relación a tres valores: honestidad, respeto y compañerismo. Los resultados sobre estos dilemas se relacionan con los estadios de Kohlberg, con el objeto de comprobar su nivel de desarrollo moral. La mayoría de los participantes están en el nivel convencional y, entre ellos, las chicas obtienen resultados más significativos en dichos valores que los chicos. Para finalizar, se establece una discusión de estos resultados con otros estudios similares.
\end{abstract}

Palabras clave: el desarrollo moral, deporte escolar en el contexto europeo, dilemas sociomorales, honestidad, respeto, compañerismo.

\begin{abstract}
School sports are an educational activity with a large participation in the national and international context. This article presents a study on moral development through social-moral dilemmas, with twelve- year-old school children involved in school sports in the European context: Spain, France, Italy and Portugal. To carry out this study a rating scale has been used for each of the dilemmas and a discussion group has been created to deliberate on three values: honesty, respect and companionship. The results associated to these dilemmas have been related to the Kohlberg's stages, in order to scrutinize their level of moral development. Most participants are in the conventional level, and among them, girls get more significant results in those values than boys. Finally, a discussion relating these results with similar studies is presented.
\end{abstract}

Key words: moral development, school sports in the European context, social-moral dilemmas, honesty, respect, companionship.

\section{RESUMO}

O esporte escolar é uma atividade educativa com grande participação no contexto nacional e internacional. Este é um estudo sobre o desenvolvimento moral através de dilemas sócio-morais, com estudantes de 12 anos, que participam no esporte escolar no contexto europeu de: Espanha, França, Itália e Portugal. Utiliza-se uma escala de valoração de cada um dos dilemas, e um grupo de discussão sobre três valores: honestidade, respeito e companheirismo. Os resultados sobre esses dilemas relacionam-se com os estágios de Kohlberg, com o objetivo de comprovar o nível de desenvolvimento moral. A maioria dos participantes está no nível convencional e, entre eles, as meninas obtêm resultados mais significativos nesses valores que os meninos. Finalmente, estabelece-se uma discussão desses resultados com outros estudos semelhantes.

Palavras-chave: Desenvolvimento moral, esporte escolar no contexto europeu, dilemas sócio-morais, honestidade, respeito, companheirismo. 


\section{INTRODUCCION}

El deporte escolar representa una práctica física orientada a la educación integral del niño. Esta actividad debe promover la participación, el juego limpio, la solidaridad, el respeto a las normas y a los compañeros de juego (Cruz et al., 1991). En estos últimos años (Fraile, 1990; Blázquez, 1995; Devís, 1994, 1996; Hernández y Velázquez, 1996; Solar, 1997; Petrus, 1998; Velázquez y Hernández, 2001; Fraile et al., 2001; Contreras, De la Torre y Velázquez, 2001; Alamo, 2001, 2004; González Carballude, 1998, 2004; Velázquez Buendía, 2004 y Ortúzar, 2004) destacan el carácter educativo de dicha práctica.

Desde una perspectiva crítica, en el deporte escolar se reproducen los valores hegemónicos en la sociedad neoliberal (Sage, 1990). Ya que se organiza y desarrolla de forma jerárquica, el entrenador impone su práctica a los jugadores; busca la eficacia y el rendimiento en la competición deportiva; discrimina a los menos capaces, etc.

Para que el deporte escolar atienda la formación integral de los escolares deberá atender los valores sociomorales desde el trabajo colaborativo, la igualdad de oportunidades, la salud (Cagigal, 1979), sin diferencias de género, edad, capacidades, intereses y culturas, así, como resolver los conflictos (Jares, 2006), (Fraile et al., 2008) y el desarrollo afectivo, emocional y social (Castañar et al., 2006).

No obstante, un tipo de práctica se viene vinculando más al sistema federativo y a la especialización deportiva a través de la competición, mientras que otra es de carácter más recreativa (Díaz Lucea, 1999). Son dos modos para interpretar el deporte escolar: Ganar como finalidad del juego o como circunstancia del juego (Fraile, 2005).

Respecto al género, destacamos el tratamiento diverso que reciben las chicas en el deporte, siendo claramente diversas en cuanto al tipo de práctica, las posibilidades de acceso, el interés y la motivación, la edad de abandono, etc. Las chicas manifiestan menores aspiraciones de éxito deportivo que los chicos, por su carácter menos competitivo (Gutiérrez, 2003). A su vez, Scraton (1995) señala que las oportunidades de realizar algún tipo de práctica deportiva y de acceder a una profesión vinculada con la actividad deportiva son restringidas para las mujeres, motivados por los cambios en los sistemas económicos, las crecientes ofertas deportivas y la creación de centros de formación para profesores especialistas.

El deporte de competición exige, cada vez más, una mayor implicación de tiempo y de capacidades para realizarlo. Esa especialización determina que esta actividad sea cada vez más selectiva. Así, aparecen claras diferencias de género respecto a la participación en el deporte de competición, donde el número de hombres duplica al de mujeres (García Ferrando, 1986, 2001).

El deporte escolar en los países europeos objeto de estudio sigue un modelo similar al español, ya que su sistema de organización, sus finalidades y características coinciden con el actual modelo federativo (Fraile et al., 2004). Será labor de los educadores diseñar un modelo de práctica que favorezca el desarrollo de los valores sociales en los escolares.

El objetivo de este estudio es: conocer qué nivel de razonamiento moral se fomenta en el deporte escolar, en el contexto europeo y a través de qué valores sociomorales se desarrolla. Mientras que en otros estudios se analizaba la presencia de los valores educativos en el deporte escolar (Fraile y De Diego, 2006). En este caso, nos centramos en tres valores como son: la honestidad, el respeto y el compañerismo, a partir de tres dilemas sociomorales relacionados con dichos valores que fueron aplicados a una muestra 
de escolares europeos (Francia, España, Italia y Portugal) de doce años participantes en el deporte escolar.

El desarrollo del razonamiento moral es un tópico al que se recurre en numerosos estudios educativos (Díaz-Aguado y Medrado, 1995; Pérez Delgado y Mestre, 1995), y que también tiene su presencia en la actividad físico-deportiva (Barba, Barba y Muriarte, 2003; Gutiérrez, 1995, 2003; Gutiérrez y Vivó, 2005).

En este estudio nos apoyaremos en la teoría del desarrollo cognitivo-evolutivo de Kohlberg (1992). A partir de Piaget (1985), se considera que en las primeras edades los niños conciben las reglas como algo dependiente de la autoridad externa; más tarde como medios para obtener recompensas y satisfacción de sus necesidades; después para obtener la aprobación y la estima social; posteriormente, como soportes de un orden ideal y, finalmente, como principios sociales necesarios para vivir. Estos principios se ven reflejados por Kohlberg (1989) en una clasificación de estadios que justifican la evolución del desarrollo moral: $1^{\circ}$ nivel Moral preconvencional (obediencia y pragmatismo); $2^{\circ}$ nivel Convencional (concordancia, Ley y Orden) y el $3^{\circ}$ nivel Postconvencional (consenso social y principios universales).

Es necesario conocer cuál es la presencia de los valores educativos en el deporte escolar y comprobar si la competición deportiva beneficia o perjudica ese desarrollo educativo (Cruz, 1987, 1997). ¿Cuál es el grado de cumplimiento de las reglas de juego, aceptación de las normas, de las decisiones de los entrenadores y de los compañeros? Valores como la honestidad, el respeto y el compañerismo deben estar presentes en este tipo de actividades (Bredemeier, et al., 1995). Los educadores y las familias deben ayudar a los escolares a descubrir y tomar conciencia de sus valores, analizando cómo pueden construir mejor su personalidad moral (De Knop, 1993). Aprender a enfrentarse a lo largo de su vida a situaciones sociomorales.

Los valores seleccionados para el estudio coinciden con los recogidos en Gutiérrez (1995; 2003, 2005); Fraile (1996, 1999); Martínez y Buxarrais (2000) y que los participantes en el deporte escolar destacan:

La Honestidad hace referencia a la integridad de una persona, es decir, a su capacidad para vivir de un modo auténtico, genuino y de acuerdo con unos valores morales. Por ello, consideramos a una persona honesta cuando actúa con rectitud y cuando piensa de forma coherente a como actúa. En el deporte, los escolares deben ser honestos, cumpliendo con unos principios éticos y morales. Deben mostrar una actitud honesta en el: respeto a las reglas del juego, participando de acuerdo a unos principios morales, cumpliendo con los compromisos, normas y obligaciones contraídas. Por último, los educadores deben cuidar que éstos respeten honestamente las reglas y las normas deportivas (Fraile, 2001).

El Respeto es una actitud y disposición a mantener una buena relación con los demás, y no subestimar o infravalorar el comportamiento de los compañeros. El respeto se relaciona con: el reconocimiento y la dignidad, así como con la atención a los derechos y deberes que uno tiene con los demás Sennett (2003). En el deporte, el respeto representa relacionarnos de forma amistosa con los demás y valorar la integridad de las personas "fair-play". Desde el compromiso con los compañeros y con el entrenador. El árbitro y el entrenador deben exigir que los jugadores respeten las reglas del juego. 
El compañerismo tiene como finalidad ayudar y mejorar el bienestar personal de todos. Evoluciona en las diferentes etapas junto con otros factores madurativos. Mientras que en las primeras edades escolares se da una similar importancia a los valores de consecución de metas que al compañerismo, después se desarrollará entre los escolares esa mayor relación grupal (Gutiérrez, 1995). Los educadores deportivos deben fomentar el compañerismo y la acción colaborativa a partir de reducir las acciones individuales. En los deportes de equipo, el aprendizaje colectivo y la cohesión son valores fundamentales para el desarrollo de las acciones tácticas ofensivas y defensivas del juego.

\section{METODO}

En este estudio los dilemas morales han sido un recurso metodológico para conocer el estadio de razonamiento sociomoral de los participantes. Hemos revisado y analizado opiniones diversas, tras un proceso de reflexión, debate y diálogo grupal. En el contexto de las actividades deportivas surgen multitud de dilemas morales vinculados a los intereses e inquietudes de los escolares (Ruiz Omeñaca, 2004).

Con el objeto de conocer: qué nivel de razonamiento moral se fomenta en el deporte escolar, hemos revisado las creencias sociomorales de los escolares y su justificación, desde los dilemas morales relativos a la honestidad, el respeto y el compañerismo. También, comprobado las diferencias de género y entre países sobre esos valores. Por último, se han relacionado esas opiniones de los escolares con los estadios de desarrollo sociomoral según Kohlberg (1989).

La muestra representativa del estudio ha estado formada por 920 escolares de 12 años de: Valladolid (España), Oporto (Portugal), Roma (Italia) y Pau-Tarbes (Francia) y, a su vez, participantes en el deporte escolar.

Tabla 1

Participantes en el estudio por países

\begin{tabular}{|l|c|c|c|c|c|}
\hline & \multicolumn{2}{|c|}{ Chicos } & \multicolumn{2}{c|}{ Chicas } & Total \\
\hline \multicolumn{1}{|c|}{ Países } & & $\%$ & & $\%$ & \\
\hline España & 104 & 46 & 122 & 54 & 226 \\
\hline Italia & 108 & 49 & 107 & 50 & 215 \\
\hline Francia & 110 & 48 & 120 & 52 & 230 \\
\hline Portugal & 126 & 51 & 123 & 49 & 249 \\
\hline & 448 & 49 & 472 & 51 & 920 \\
\hline
\end{tabular}

La selección de la muestra se realizó a partir de los principios de incidentalidad, voluntariedad y conglomerado de los colegios participantes (como método de muestreo simple, por el que todos los elementos que constituyen la población se encuentran dentro de ese conglomerado, y por tanto tienen la misma posibilidad de ser elegidos), en este caso: tener 12 años y participar en el deporte escolar. 
Una vez redactados y validados los dilemas morales fueron aplicados en los diferentes centros escolares seleccionados en las localidades de estudio. En la primera fase el educador como dinamizador tenía como labor: Organizar el grupo y presentarles cada uno de los dilemas, dejando un tiempo para que cada uno de forma individual diera su respuesta, así como comprobar si todos los participantes habían entendido bien el dilema y elegían una de las dos opciones presentadas por cada dilema. En la segunda fase utilizamos los grupos de discusión a partir de los criterios de Krueger (1991) y Callejo (2001), en donde: a) Los representantes de todos los colectivos favorecían la heterogeneidad; b) El número de escolares no superaba la decena; c) La duración no fue superior una hora.

Por último, el tratamiento de la información estadística (cuantitativa) y naturalista (cualitativa) fue realizada en el primer caso a partir del paquete estadístico S.P.S.S. en su versión 9.0. Y, en el segundo, desde el programa Nudits Vivo 11.

\section{RESULTADOS}

A continuación recogemos la valoración que los escolares hacen de los dilemas sociomorales: honestidad, respeto y compañerismo. Son analizados en relación al género, por países y su conexión con los estadios de Kohlberg (1989).

\subsection{DILEMA SOBRE LA HONESTIDAD: INCUMPLIR LAS REGLAS PARA OBTENER VENTAJA EN EL RESULTADO}

Es el partido más importante de la temporada. Tu equipo está perdiendo y falta poco para concluir. Tienes la oportunidad de simular un penalti y si el árbitro lo sanciona el equipo puede conseguir un mejor resultado.

A: Lo haría, es una situación normal del juego.

B: No lo haría, es una conducta deshonesta.

Tabla 2

Valoraciones de los escolares sobre la honestidad

\section{PREGUNTAS PARA EL GRUPO DE DISCUSION}

Justifica tu respuesta

¿Consideras que se pueden hacer trampas en el juego, siempre que se pueda, para obtener una ventaja para uno mismo o para el equipo? ¿Qué es más importante, ser honesto o vencer?

\begin{tabular}{|l|c|c|c|c|c|c|c|}
\hline & & \multicolumn{2}{|c|}{ Género } & \multicolumn{4}{c|}{ Países } \\
\hline Alternativas & $\begin{array}{c}\text { Participantes } \\
\mathrm{n}=916\end{array}$ & $\begin{array}{c}\text { Chicos } \\
\mathrm{n}=447\end{array}$ & $\begin{array}{c}\text { Chicas } \\
\mathrm{n}=469\end{array}$ & $\begin{array}{c}\text { España } \\
\mathrm{n}=226\end{array}$ & $\begin{array}{c}\text { Italia } \\
\mathrm{n}=215\end{array}$ & $\begin{array}{c}\text { Francia } \\
\mathrm{n}=230\end{array}$ & $\begin{array}{c}\text { Portugal } \\
\mathrm{n}=245\end{array}$ \\
\hline A) Deshonestidad & $46 \%$ & $62 \%$ & $36 \%$ & $47 \%$ & $51 \%$ & $47 \%$ & $52 \%$ \\
\hline B) Honestidad & $54 \%$ & $38 \%$ & $64 \%$ & $53 \%$ & $49 \%$ & $53 \%$ & $48 \%$ \\
\hline
\end{tabular}


En los resultados de este dilema sobre la honestidad, algo más de la mitad de los escolares (54\%) están de acuerdo con la Opción B (honestidad); mientras que el (46\%) están a favor de la Opción A (deshonestidad), lo cual representa que algunos más de la mitad de los escolares no simularían un penalti, por considerarlo deshonesto, mientras que el resto, un porcentaje algo menor, sí lo harían, adoptando una postura antideportiva.

\section{Tabla 3}

Diferencias estadísticamente significativas sobre la honestidad

\begin{tabular}{|l|c|c|c|c|c|c|c|c|c|c|c|}
\hline Alternativas & Part. & Sig. & Chicos & Chicas & Sig. & España & Portugal & Sig. & Francia & Portugal & Sig. \\
\hline A) Deshonestidad & $46 \%$ & $1 \%$ & $62 \%$ & $36 \%$ & $1 \%$ & $47 \%$ & $52 \%$ & $5 \%$ & $47 \%$ & $52 \%$ & $5 \%$ \\
\hline B) Honestidad & $54 \%$ & $1 \%$ & $36 \%$ & $64 \%$ & $1 \%$ & $53 \%$ & $48 \%$ & $5 \%$ & $53 \%$ & $48 \%$ & $5 \%$ \\
\hline
\end{tabular}

En tabla anterior sobre la honestidad existe una diferencia estadísticamente significativa del $1 \%$ entre (54\%) de los participantes favorables de adoptar una actitud honesta, frente $(46 \%)$ que no les importa engañar para ganar. Se produce una diferencia significativa a favor de las chicas (64\%) sobre los chicos (38\%). Los deseos de ganar de los escolares puede ser una de las razones de hacerles tomar esa postura no deportiva (deshonesta) fingiendo una acción de penalti. Sin embargo, la mayoría de las chicas rechazan ese comportamiento deshonesto. Son menos las diferencias significativas entre los escolares españoles $(53 \%)$ y portugueses $(48 \%)$.

a) Nivel convencional. Estos resultados se relacionan con el nivel de moralidad a partir de respetar las normas sociales (deportivas). Destacan aquellos escolares que actúan sin engañar con objeto de cumplir con las normas de orden social y las conductas éticas a respetar por un equipo. No es ético simular ninguna acción para beneficiarse en el resultado. El grado de honestidad es mayor en las chicas (Opción B) indicando un mayor desarrollo moral, relacionado con las actitudes y valores deportivos:

"no debemos actuar de forma antideportiva cuando un equipo se merece ganar porque es superior". "No nos gustaría que el contrario simulara esa acción para ganar", "si los otros no lo hacen, nosotros no tenemos que hacerlo".

Los chicos que eligen la Opción B expresan lo siguiente:

"Yo no simularía penalti, ya que eso es deshonesto y lo importante del juego es no hacer trampas". "Un equipo que se considera el mejor tiene que actuar con honestidad y deportividad, ya que si lo hace con trampas o infringiendo el reglamento entonces no es tan bueno". "El mejor equipo tiene que ganar sin necesidad de engañar o hacer trampas".

La honestidad deportiva se muestra en los escolares que actúan correctamente (cumplen con las reglas), sin considerar lo que hagan los demás. Este sentido deportivo ético les lleva a actuar por convencimiento y porque, para ellos, la honestidad debe ser un principio moral de un equipo que quiere conseguir mejores resultados deportivos, a la vez que mejorar como personas. 
b) Nivel preconvencional: En este segundo nivel la moralidad se rige por el cumplimiento de las reglas externas. Son los escolares que actúan dependiendo de la autoridad. En este caso, la opción de hacer trampas está condicionada por si el árbitro les ve o no; es decir, no es una situación marcada por su propia moral. Algunos justifican las trampas por el hecho de ganar en una competición en donde el resultado tiene gran trascendencia (ganar un título), o lo vinculan a la mera obtención de puntos con la victoria.

"Si es una final haría trampas para ganar, porque lo importante es la victoria, pero en un partido normal no sería necesario". "Simularíamos el penalti porque necesitamos los puntos y debemos hacer cualquier cosa para obtenerlos", "Si el objetivo es ganar la trampa se puede hacer".

Igualmente, en este mismo nivel preconvencional están los escolares que lo justifican (respuesta deshonesta) al no considerarla como un acto grave. Es una situación ordinaria y poco trascendente de su vida diaria. Lo relacionan con los escolares que reproducen el deporte de adultos. También están los que actúan no tanto por su motivación interna, sino como evitación del castigo.

"Simular una falta llega a ser algo habitual, ya que lo vemos en el deporte de competición, donde cada jugador juega al límite de las reglas con el hecho de ganar"." Si no me ve el árbitro simulo la falta".

Por tanto, aunque hay una actitud honesta entre todos los participantes las chicas actúan con mayor ética que los chicos. Los que están a favor de una postura honesta consideran que se debe evitar hacer trampas en el juego, actuar con deportividad, cumplir con el reglamento, ganar sin engañar. Es decir, actuar con honestidad por convencimiento y no dependiendo de ser observado por el árbitro. Mientras, entre los deshonestos se justifica ese tipo de conductas deportivas antirreglamentarias por la necesidad de ganar y por ser habituales en el deporte entre los adultos.

\subsection{DILEMA SOBRE EL RESPETO: LA ENTRENADORA DEBE RESPETAR SUS COMPROMISOS CON LA JUGADORA}

"Este año Sara ha empezado a jugar al balonmano. Se ha esforzado y progresa mucho. Hoy juega su primera competición. La entrenadora ha comentado que durante el partido dará oportunidades a todas las jugadoras del equipo. Para ella participar es más importante que el resultado. Pasan los minutos y Sara sigue en el banquillo. La entrenadora percibe que Sara es la única que no ha jugado y debería cambiarla por Marta, pero el otro equipo está remontando. Si cambia a Marta que es muy buena, se puede perder el partido".

A: La entrenadora debe hacer jugar a Sara porque es su compromiso y por respeto hacia ella.

B: Si yo fuese la entrenadora no haría jugar a Sara, se podría perder y al final el resultado es

lo más importante. Ya habrá otras oportunidades.

\begin{tabular}{|l|}
\hline PREGUNTAS PARA EL GRUPO DE DISCUSIÓN \\
\hline Justifica tu respuesta. \\
\hline Si fueses la jugadora beneficiada por seguir jugando, ¿qué harías? \\
\hline ¿Qué es más importante, participar o ganar? \\
\hline
\end{tabular}




\section{Tabla 4}

Valoraciones de los escolares sobre el respeto

\begin{tabular}{|l|c|c|c|c|c|c|c|}
\cline { 3 - 8 } \multicolumn{2}{c|}{} & \multicolumn{2}{c|}{ Género } & \multicolumn{4}{c|}{ Países } \\
\hline Alternativas & $\begin{array}{c}\text { Participantes } \\
\mathrm{n}=916\end{array}$ & $\begin{array}{c}\text { Chicos } \\
\mathrm{n}=447\end{array}$ & $\begin{array}{c}\text { Chicas } \\
\mathrm{n}=469\end{array}$ & $\begin{array}{c}\text { España } \\
\mathrm{n}=226\end{array}$ & $\begin{array}{c}\text { Italia } \\
\mathrm{n}=215\end{array}$ & $\begin{array}{c}\text { Francia } \\
\mathrm{n}=230\end{array}$ & $\begin{array}{c}\text { Portugal } \\
\mathrm{n}=245\end{array}$ \\
\hline A) Respeto & $80 \%$ & $78 \%$ & $83 \%$ & $85 \%$ & $86 \%$ & $77 \%$ & $81 \%$ \\
\hline B) No respeto & $20 \%$ & $22 \%$ & $17 \%$ & $15 \%$ & $14 \%$ & $23 \%$ & $19 \%$ \\
\hline
\end{tabular}

En el segundo de los dilemas apreciamos una mayor tendencia hacia la Opción A $(80 \%)$ sobre la Opción B (20\%). Los escolares valoran que la entrenadora haga jugar a Sara, ese fue su compromiso y por respeto hacia ella.

\section{Tabla 5}

Diferencias estadísticamente significativas sobre el respeto

\begin{tabular}{|l|c|c|c|c|c|c|c|c|c|c|c|}
\hline Alternativas & Part. & Sig. & Chicos & Chicas & Sig. & España & Portugal & Sig. & Francia & Portugal & Sig. \\
\hline A) Respeto & $80 \%$ & $1 \%$ & $78 \%$ & $83 \%$ & $1 \%$ & $85 \%$ & $77 \%$ & $5 \%$ & $86 \%$ & $77 \%$ & $5 \%$ \\
\hline B) No respeto & $20 \%$ & $1 \%$ & $22 \%$ & $17 \%$ & $1 \%$ & $5 \%$ & $23 \%$ & $5 \%$ & $14 \%$ & $23 \%$ & $5 \%$ \\
\hline
\end{tabular}

Existe diferencia estadísticamente significativa (80\%) contra $(20 \%)$ a favor del respeto. En las chicas es mayor ese respeto (83\%) que en los chicos (78\%). El espíritu competitivo de los chicos les lleva a tomar una postura menos deportiva que las chicas. Menos diferencias significativas hay entre los escolares españoles $(85 \%)$ y los franceses $(77 \%)$ y entre los escolares italianos (86\%) sobre los franceses $(77 \%)$.

Que las chicas valoren algo más que los chicos que la entrenadora respete sus compromisos con Sara refleja el mayor interés por participar que por ganar, están a favor de que Sara juegue a pesar de poder perder.

a) Nivel convencional. La mayoría de los escolares están en el nivel convencional, al estar de acuerdo con que la entrenadora cumpla con su compromiso (sacando a esa jugadora) por encima del propio resultado deportivo, corresponde con unos principios y valores morales. Ese mayor respeto de las chicas se refleja en las siguientes opiniones:

"Que la entrenadora se comprometa con una jugadora para hacerla participar es más importante que el resultado". "Sara también tiene derecho a participar". "Después de lo que lleva entrenado, Sara tiene derecho a jugar un poco de tiempo". "Sara debe ir cogiendo confianza como jugadora".

b) Nivel preconvencional. Algunos destacan la necesidad de ganar (espíritu competitivo) sobre el respeto del compromiso con Sara, ya que no es buena jugadora. Prefieren 
que la entrenadora no cumpla su compromiso, ya que pueden perder. Deben jugar solo las mejores y en partidos posteriores, con rivales inferiores, dar a todos esa oportunidad de jugar.

"Lo primero y más importante es el resultado, luego que jueguen todos". "La entrenadora no debe retirar a una de las mejores jugadoras". "En partidos menos importantes pueden jugar los malos".

Se muestran dos perspectivas, una a favor y otra en contra de respetar la decisión de la entrenadora y, con ello, dar o no prioridad al resultado sobre la participación. Son dos visiones que tradicionalmente se ven en el deporte escolar. La primera (mayoritaria) menos competitiva, que aboga por respetar a la entrenadora que hace participar a la jugadora menos buena. La otra opción (minoritaria), competitiva, representa al deporte federado y donde prima el resultado sobre la participación.

\subsection{DILEMA SOBRE EL COMPAÑERISMO: UN CHICO MUESTRA COMPAÑERISMO HACIA UNA NIÑA QUE SE QUEDA SIN EQUIPO}

Tabla 6

Valoración de los escolares sobre el compañerismo

"Hoy la entrenadora deja que los escolares se organicen en grupos para jugar. Varios amigos se
juntan en uno de ellos. Es su oportunidad para compartir el mismo grupo, porque la entrenadora
habitualmente no los deja hacerlo. Claudia por ser la menos habilidosa del equipo se ha quedado
sin grupo. La entrenadora pide que algún grupo la acoja. Miguel se ofrece a invitarla. No le
parece justo que Claudia se quede sola, aunque a sus amigos no les gustará la idea.

A: Si yo fuese Miguel invitaría a Claudia a formar parte de mi grupo, es muy triste dejarte fuera de un grupo.

B: Si yo fuese Miguel no diría nada para no tener problemas con los amigos. También puede formar parte de otro grupo.

\section{PREGUNTAS PARA EL GRUPO DE DISCUSION}

Justifica tu respuesta.

Que Miguel trate de acoger a Claudia en su grupo es una buena conducta, ¿por qué?

Saber que a sus amigos no les va a gustar nada que Claudia vaya con ellos, puede condicionar la decisión que tome Miguel, ¿por qué?

\begin{tabular}{|l|c|c|c|c|c|c|c|}
\cline { 3 - 8 } \multicolumn{2}{c|}{} & \multicolumn{2}{c|}{ Género } & \multicolumn{4}{c|}{ Países } \\
\hline Alternativas & $\begin{array}{c}\text { Sujetos } \\
\mathrm{n}=916\end{array}$ & $\begin{array}{c}\text { Chicos } \\
\mathrm{n}=447\end{array}$ & $\begin{array}{c}\text { Chicas } \\
\mathrm{n}=469\end{array}$ & $\begin{array}{c}\text { España } \\
\mathrm{n}=225\end{array}$ & $\begin{array}{c}\text { Italia } \\
\mathrm{n}=215\end{array}$ & $\begin{array}{c}\text { Francia } \\
\mathrm{n}=230\end{array}$ & $\begin{array}{c}\text { Portugal } \\
\mathrm{n}=245\end{array}$ \\
\hline A) Compañerismo & $91 \%$ & $88 \%$ & $94 \%$ & $91 \%$ & $95 \%$ & $90 \%$ & $89 \%$ \\
\hline B) No compañerismo & $9 \%$ & $12 \%$ & $6 \%$ & $9 \%$ & $5 \%$ & $10 \%$ & $11 \%$ \\
\hline
\end{tabular}


En este último dilema la opción A es claramente la más valorada (91\%), aprobando la actitud de Miguel de compañerismo, al invitar a Claudia a formar parte del equipo, evitando que se quede sin participar. La opción B es rechazada por la mayoría de los escolares $(9 \%)$.

\section{Tabla 7}

Diferencias estadísticamente significativas sobre el compañerismo

\begin{tabular}{|l|r|c|c|c|c|c|c|c|c|c|c|}
\cline { 2 - 11 } \multicolumn{1}{c|}{} & Part. & Sig. & Chicos & Chicas & Sig. & Italia & Francia & Sig. & Italia & Portugal & Sig. \\
\hline A) Compañerismo & $91 \%$ & $1 \%$ & $88 \%$ & $94 \%$ & $1 \%$ & $95 \%$ & $90 \%$ & $5 \%$ & $95 \%$ & $89 \%$ & $5 \%$ \\
\hline B) No compañerismo & $9 \%$ & $1 \%$ & $12 \%$ & $6 \%$ & $1 \%$ & $5 \%$ & $10 \%$ & $5 \%$ & $5 \%$ & $11 \%$ & $5 \%$ \\
\hline
\end{tabular}

Destacan los partidarios del compañerismo (91\%) sobre el resto (9\%). Hay diferencias significativas entre chicas $(94 \%)$ y los chicos $(88 \%)$, que muestran un mayor espíritu competitivo y menor compañerismo. Igualmente, entre los escolares italianos (95\%) sobre los franceses $(90 \%)$ y de esos italianos sobre los portugueses $(89 \%)$.

a) Nivel convencional. A este nivel pertenece la mayoría que apoya el compañerismo de Miguel. Se relaciona con un firme principio moral de amistad hacia Claudia. La mayoría de los escolares, chicas, consideran que Miguel hace bien acogiendo a Claudia para que juegue.

"Los buenos amigos entienden que Miguel invite a participar a Claudia para que no se quede sola". "Miguel debe invitar a Claudia porque es importante conocer a otras personas, sin preocuparle lo que opinen los demás".

En este primer nivel también están los que consideran que Miguel debería convencer al grupo sobre la necesidad de ser solidarios con esa compañera que está sin equipo, y no actuar sólo pensando en el rendimiento.

"Yo trataría de convencer a los compañeros sobre la necesidad de ser compañeros, ya que alguno de nosotros también podía quedar solo en otra situación".

b) Nivel preconvencional. En este nivel están los pocos escolares que adoptan una postura egoísta, dando prioridad al grupo cerrado y, por tanto, no aceptan compartir con alguien (una chica), no les preocupa que ésta pueda sentirse discriminada.

"Yo no la acogía, pues no hay muchos momentos en los que en el grupo podamos estar solos".

Otros consideran que admitir a una chica puede influir negativamente en los resultados. Sólo cuando la chica es buena jugadora se la invita a participar, mientras que si no lo es no se contará con ella. Hay una postura interesada y no tan solidaria, ya que su admisión dependerá de sus capacidades y de lo que pueda aportar a nivel del grupo.

"Podemos en principio acogerla en el grupo y esperar a cómo juega para que siga o no con nosotros". 
Otros prefieren dejar la responsabilidades al entrenador.

"El entrenador debería tomar esa decisión de evitar que alguien del grupo se quede solo cuando los grupos están ya constituidos". "Si el motivo de rechazarla es su baja habilidad, el entrenador debería organizar los equipos, evitando que nadie quede fuera".

La mayoría de los escolares (chicas) están a favor del compañerismo y, por tanto, valoran que Miguel invite a Claudia al equipo. A pesar del espíritu competitivo de los chicos, en este caso, se acepta que una chica forme parte del equipo, aunque pueda perjudicar el resultado. Para otros, depende del nivel de la jugadora o pasan la responsabilidad al entrenador que se debe adelantar a la opinión del grupo.

\section{DISCUSIÓN}

A continuación comentaremos cómo se relacionan los resultados con los objetivos inicialmente previstos. ¿Cuáles son las posibles razones que han llevado a los escolares a adoptar unas $\mathrm{u}$ otras posturas ante los diferentes dilemas sociomorales representativos de los valores de honestidad, respeto y compañerismo?

a) La honestidad. Algo más de la mitad de los escolares se muestran a favor de la honestidad; es decir, prefieren no engañar al árbitro (simulando el penalti), a pesar de que con ello podían ganar el partido. Esa actitud honesta es mayor entre las chicas (Scraton, 1995; Fraile y De Diego, 2006). Los deseos de ganar de los chicos les llevan a estar a favor de fingir el penalti (deshonesta).

Según los estadios de Kohlberg (1989), la mayoría de los escolares estarían en un nivel convencional. Estos escolares consideran que en el deporte y en la vida no se deben realizar trampas, por convencimiento moral y porque esto es un indicador de los buenos equipos.

Aquellos escolares del nivel preconvencional relativizan el engaño deportivo y destacan que el resultado es lo importante. Engañar cuando el árbitro no te ve es habitual en la competición de adultos y forma parte del entrenamiento oculto. Incluso, cuando hay algún jugador que lo recrimina es discriminado por el resto de los compañeros.

Bredemeier et al. (1995) consideran que no siempre en el deporte escolar se fomenten valores como: la deportividad, el respeto al contrario y a las reglas de juego. Así, los educadores deportivos deben educar esos valores, priorizando aquellas buenas actuaciones morales sobre los resultados deportivos.

\subsection{EL RESPETO}

La mayoría de los escolares (en su mayoría chicas) valoran que la entrenadora respete el compromiso adquirido con la jugadora, haciéndola jugar: a) Aunque tenga menos capacidades deportivas que el resto, debe participar en la competición, sin valorar el resultado; b) La entrenadora ya se había comprometido con ella; c) Por último, algunos escolares dan prioridad al resultado, considerando que deben competir sólo los mejores. 
El respeto se representa por el tratamiento digno de la entrenadora hacia la jugadora y de los jugadores hacia la decisión de la entrenadora. Para Escartí (2005), en el deporte se debe crear un ambiente educativo de respeto hacia los participantes. Así como evitar la discriminación por razón de género, nivel de competencia motriz, procedencia cultural, etc. Destacamos el carácter menos competitivo de las chicas (Fraile et al., 2001). Por tanto, los educadores deben exigir respeto entre los escolares.

\subsection{EL COMPAÑERISMO}

La mayoría de los escolares (chicas) se muestran a favor del compañerismo y, por tanto, aprueban que Miguel invite a Claudia para que forme parte del equipo. El compañerismo es uno de los valores más relevantes en el equipo (nivel convencional). Las chicas son solidarias con una compañera reclamando que forme parte del equipo. Otros (nivel preconvencional) dan prioridad a ganar antes de que una chica se integre en el equipo, ya que puede perjudicar el resultado. Sólo la aceptarían si destacara como jugadora. Por último, están los que dejan estas decisiones a la entrenadora.

El compañerismo como una de las claves de la actividad deportiva se relaciona con la interacción, la cohesión grupal, la amistad y con el trabajo colectivo que se desarrolla en el deporte educativo. Gutiérrez (1995) destaca en la edad escolar el compañerismo sobre aquellas conductas egoístas que buscan el resultado finalista y personal. La opinión de los escolares puede verse afectada, ya que el deseo de ganar les lleva a justificar ciertas conductas (dejar a una compañera sin participar). En este caso, los escolares muestran un alto compañerismo respecto a la jugadora, integrándola en el equipo sin preocupar el resultado de la competición.

Para finalizar, la discusión sobre los dilemas morales ha supuesto un buen recurso metodológico para conocer cómo se origina y desarrolla la educación moral entre los escolares, ya que les ha exigido comunicarse y reflexionar, así como identificar los principios de actuación en situaciones conflictivas y ante valores enfrentados (Cantillo et al., 2005).

Aunque Bredemeier y Shields (1986) consideran que los escolares ocupan niveles bajos de moralidad en el deporte (cuando utilizan la agresión). En este estudio, la mayoría de los escolares (preferentemente chicas) adoptan un nivel convencional en sus respuestas respecto a los valores de honestidad, respeto y compañerismo. Drewe (1999), en un estudio sobre dilemas éticos y moralidad establece seis categorías morales: (1) honestidad con los demás; (2) imparcialidad/justicia; (3) respeto hacia los otros; (4) no agresión; (5) respeto para el juego; (6) miedo a no perder. De estas categorías se recogen las tres primeras en este estudio.

Por último, para Siedentop (2002) la mejora del carácter en los escolares no es automática gracias al deporte. El desarrollo sociomoral debe ayudar a esa mejora, siendo un objetivo prioritario para los educadores deportivos e investigadores que evalúan los programas deportivos y sus efectos (Jones y McNamee, 2000; Kirk, 2006).

\section{CONCLUSIONES}

Más de la mitad de estos escolares adoptan una actitud honesta (especialmente las chicas), al considerar que no se deben buscar ventajas en el juego simulando una falta para que el árbitro señale penalti y obtener un mejor resultado. 
La mayoría de estos escolares (chicas) destacan el valor del respeto y valoran que la entrenadora cumpla su compromiso de hacer participar a la jugadora con menos capacidades deportivas.

La mayoría de estos escolares (chicas) están de acuerdo con el valor de compañerismo, ya que aprueban que uno de los jugadores quiera incorporar a su equipo a una jugadora a formar parte del grupo.

La evolución del desarrollo moral en los escolares que participan en el deporte escolar se relaciona con los niveles y estadios establecidos por Kohlberg. De forma prioritaria con el nivel convencional, ya que la mayoría adoptan una postura favorable con los dilemas que se presentan.

\section{BIBLIOGRAFIA}

Alamo, J.M. (2001). Análisis del deporte escolar en la Isla de Gran Canaria. Hacia un modelo de deporte escolar. Tesis doctoral. Universidad de Las Palmas de Gran Canaria.

Alamo, J.M. (2004). El deporte escolar en Canarias. En Fraile (coord.) y Otros. El deporte escolar en el siglo XXI: análisis y debate desde una perspectiva europea. Barcelona. Graó.

Barba, J., Barba, J.A. y Muriarte, D. (2003). Desarrollo moral en educación física. Una propuesta de dilemas morales a través de la EF y el deporte. EF deporte, 61, http://www.efdeportes. com/efd61/moral.htm.

Blázquez, D (1995). La Iniciación Deportiva y el Deporte Escolar. Barcelona. Inde.

Bredemeier, B.J., Bostrom, D.E., Gardner, D.E., Light, D.L. (1995) ."Leadership, Cohesion, and Team Norms Regarding Cheating and Aggression”. Sociology of Sport Journal, No 12, 324-336.

Bredemeier, B.J., Shields, D.L. (1986). Game reasoning and interactional morality. Journal of Genetic Psychology 147(2), 257-275.

Cagigal, J.M. (1979). Cultura intelectual y cultura física. Buenos Aires. Kapelusz.

Callejo, J. (2001). El grupo de discusión: introducción a una práctica de investigación. Barcelona. Ariel.

Cantillo, J., Domínguez, A., Encinas, S., Muñoz, A., Navarro, F. y Salzar, A. (2005). Dilemas morales. Un aprendizaje de valores mediante el diálogo. Valencia. Nau Libres.

Castañer, M. (Coord.), Grasso, A.E., López, C., Mateu, M, Motos, T. y Sánchez, R. (2006). La inteligencia corporal. Barcelona. Graó.

Contreras, O., De La Torre, E. y Velázquez, R. (2001). Iniciación deportiva. Madrid. Síntesis.

Cruz, J. (1987). Aportaciones a la iniciación deportiva. Apuntes, 9.

Cruz, J. (1997). Psicología del deporte. Madrid. Síntesis.

Cruz, J. et al. (1991). "Identificación de valores relevantes en jugadores jóvenes de fútbol". Revista de Investigación y Documentación sobre las Ciencias de la Educación Física y el Deporte $\mathrm{N}^{\mathrm{o}} 19,81-89$.

De Knop, P. (1993). El papel de los padres en las práctica deportiva infantil. Málaga. Unisport.

Devis, J. (1994). "Deporte, educación y sociedad: hacia un deporte escolar diferente". Revista de Educación $\mathrm{N}^{\mathrm{o}} 306,455-472$.

Devis, J. (1996). Educación Física, deporte y currículum. Madrid. Visor.

Díaz-Aguado, M.J. y Medrano, C. (1995). Educación y razonamiento moral. Bilbao. Mensajero.

Díaz-Lucea, J. (1999). La enseñanza y aprendizaje de las habilidades y destrezas motrices básicas. Barcelona, INDE.

Drewe, S.B. (1999). Moral reasoning in sport: Implications for physical education. Sport, Education \& Society 4(2), 117-130.

Escartí, A. (coord.), Pascual, C. y Gutiérrez, M. (2005). Responsabilidad a través de la Educación Física y el Deporte. Barcelona. Graó. 
Fraile Aranda, A. (1990). "Un modelo didáctico para un programa de escuelas deportivas". Perspectivas de la actividad física y el deporte, 4, 33-36.

Fraile Aranda, A. (1996). Reflexiones sobre la presencia del deporte en la escuela. Rev. Educación Física 64, 5-11.

Fraile Aranda, A. (1999). Informe del estudio sobre el diseño y evaluación de un programa de actividad física recreativa en edad escolar: Un estudio de casos sobre las experiencias de Valladolid y Segovia. Junta de Castilla y León (no publicado).

Fraile Aranda, A. (2001). La competición en el deporte escolar como factor segregador. En M. Latiesa, Deporte y Cambios social en el Umbral del Siglo XXI. Investigación Social y Deporte, 5, 395-411. Madrid. Esteban Sanz.

Fraile Aranda, A. (coord.), De Diego, R., Monjas, R., Gutiérrez, S. y López, V. (2001). Actividad física jugada. Una propuesta educativa para el deporte escolar. Marfil. Alcoy.

Fraile Aranda, A. (coord.), Aguado, P., Díez, M., Fernández, M., Frutos, M., De Frutos, S., Pérez, L. y Romo, C. (2003). Actividad física y salud en Educación Secundaria. Junta de Castilla y León.

Fraile Aranda, A. (coord.) y Otros (2004). El deporte escolar en el siglo XXI: análisis y debate desde una perspectiva europea. Barcelona. Graó.

Fraile Aranda, A. y De Diego, R. (2006). Motivaciones de los escolares europeos para la práctica del deporte escolar. Un estudio realizado en España, Italia, Francia y Portugal. Revista Internacional de Sociología (RIS), 44, 85-109.

Fraile Aranda, A. (coord.). López, V.M., Ruiz, J. y Velázquez, C. (2008). La resolución de los conflictos en y a través de la educación física. Barcelona. Graó.

García Ferrando, M. (1986). "Un único modelo, El deporte de competición”. Apuntes de E.F. 3.

García Ferrando, M. (2001). Los españoles y el deporte: prácticas y comportamientos en la última década del siglo XX. Madrid. CSD.

Fraile Aranda, A. (1998). Organización del deporte extraescolar en los centros docentes de Barcelona. Tesis doctoral, Universidad de Barcelona.

Fraile Aranda, A. (2004). El deporte escolar en Cataluña. En Fraile (coord.) y otros. El deporte escolar en el siglo XXI: análisis y debate desde una perspectiva europea. Barcelona. Graó.

Gutiérrez Sanmartin, M. (1995). Valores sociales y deporte. Madrid. Gymnos.

Gutiérrez Sanmartin, M. (2003). Manual sobre valores en la Educación Física y el deporte. Barcelona. Paidós.

Gutiérrez Sanmartin, M. (2005). El deporte y la actividad física como herramientas para el desarrollo psicológico y social de la infancia y en la adolescencia. En Escartí, A. (coord.). Pascual, C. y Gutiérrez, M. Responsabilidad personal y social a través de la Educación Física y el deporte, 13-28. Barcelona. Graó.

Gutiérrez, M. y Vivó, P. (2005). Enseñando razonamiento moral en las clases de EF escolar. European Journal of Human Movement, 14, 1-22.

Jares, X.R. (2006). Pedagogía de la convivencia. Barcelona. Graó.

Jones, C., Mcnamee, M. (2000). Moral reasoning, moral action, and the moral atmosphere of sport. Sport Education and Society 5(2), 131-146.

Hernández, J. L. y Velázquez, R. (1996). La actividad física y deportiva extraescolar en los centros educativos. Madrid. MEC.

Kirk, D. (2006). Sport education, critical pedagogy, and learning theory: Toward an intrinsic justification for physical education and youth sport. Quest, 58(2), 255-264.

Kohlberg, L. (1989). Estadios morales y moralización. Enfoques cognitivos-evolutivo, en Turiel, E. y otros (comps.). El mundo social de la mente infantil. Madrid. Alianza.

Kohlberg, L. (1992). Psicología del desarrollo moral. Bilbao. Descleé de Brouwer.

Kueger, R.A. (1991). El grupo de discusión: una práctica para la investigación aplicada. Madrid. Pirámide. 
Martínez, M. y Buxarrais, M. R. (2000). Los valores de la educación física y el deporte en edad escolar. Aula de Innovación educativa, 91, 6-9.

Ortúzar. I. (2004). El deporte escolar y el profesorado de Educación Física. Tesis doctoral. UPV. Bilbao.

Pérez Delgado, E. y Mestre, V. (1995). El crecimiento moral. Programas socioeducativos y su eficacia en el aula. Valencia. Universidad de Valencia.

Petrus, A. (1998). Deporte escolar, en desafíos del deporte ante el III milenio: Vitoria-Gasteiz.

Ruiz Omeñaca, J. V. (2004). Pedagogía de los valores en la actividad física. Madrid. CCS.

Sage, G. H. (1990). Power and ideology in American Sport. Champaign Illinois. Human Kinetics.

Scraton, S. (1995). Educación Física de las niñas. Morata. Madrid.

Sennett, R. (2003). El respeto. Barcelona. Anagrama.

Siedentop, D. (2002). Junior sport and the evolution of sport cultures. Journal of Teaching in Physical Education, 21(4), 392-401.

Solar, L. (1997). ¿Quién nos enseña a competir? Aula de Innovación Educativa, 68 (11-13).

Velázquez, R. y Hernández, R. (2001). Deporte, práctica deportiva y formación de actitudes y valores. II Congreso de Ciencias de la Actividad física y del deporte. Universidad de Valencia.

Velázquez Buendía, R. (2004). Debate sobre algunos tópicos del deporte escolar español. En Fraile (coord.) y otros. El deporte escolar en el siglo XXI: análisis y debate desde una perspectiva europea. Barcelona. Graó. 
